\title{
Acute Promyelocytic Leukemia after whole Brain Irradiation of Primary Brain Lymphoma in AN HIV-Infected Patient
}

\author{
A. Boban ${ }^{1}$, I. Radman 1 , R. Zadro 2 , K. Dubravcic 2 , T. Maretic ${ }^{3}$, R. Civljak 3 , M. Lisic ${ }^{3}$ J. Begovac 3 \\ ${ }^{1}$ Division of Hematology, Department of Internal Medicine, Clinical Hospital Center Zagreb, Zagreb, Croatia \\ ${ }^{2}$ Clinical Institute of Laboratory Diagnostics, Clinical Hospital Center Zagreb, Zagreb, Croatia \\ ${ }^{3}$ University Hospital for Infectious Diseases, Zagreb, Croatia
}

\begin{abstract}
The occurrence of acute promyelocytic leukemia (APL) in HIV-infected patients has been reported in only five cases. Due to a very small number of reported HIV/APL patients who have been treated with different therapies with the variable outcome, the prognosis of APL in the setting of the HIV-infection is unclear. Here, we report a case of an HIV-patient who developed APL and upon treatment entered a complete remission. A 25-years old male patient was diagnosed with HIV-infection in 1996, but remained untreated. In 2004, the patient was diagnosed with primary central nervous system lymphoma. We treated the patient with antiretroviral therapy and whole-brain irradiation, resulting in complete remission of the lymphoma. In 2006, prompted by a sudden neutropenia, we carried out a set of diagnostic procedures, revealing APL. Induction therapy consisted of standard treatment with all-trans-retinoic-acid (ATRA) and idarubicin. Subsequent cytological and molecular analysis of bone marrow demonstrated complete hematological and molecular remission. Due to the poor general condition, consolidation treatment with ATRA was given in March and April 2007. The last follow-up 14 months later, showed sustained molecular APL remission. In conclusion, we demonstrated that a complete molecular APL remission in an HIV-patient was achieved by using reduced-intensity treatment.
\end{abstract}

Key words: HIV, acute promyelocytic leukemia, primary central nervous system lymphoma

\section{TO THE EDITOR}

When compared with the general population, the incidence of acute myeloid leukemia in HIV-infected patients is increased approximately twofold [1]. However, occurrence of acute promyelocytic leukemia (APL) has so far been reported in only five HIV-infected patients. [1, 2, 3, 4] Here, we report a case of an HIV-patient who developed APL and upon treatment entered a complete remission.

A 25-year-old bisexual, Caucasian male patient was diagnosed with HIV infection in 1996 . He was still antiretroviral naïve in April 2004 when primary central nervous system lymphoma (PCNSL) was diagnosed by brain biopsy. We treated the patient with HAART (highly active antiretroviral treatment) consisting of zidovudine, lamivudine and lopinavir/ritonavir, and whole brain irradiation with 46 Gy. The therapy result- ed in durable remission of the lymphoma. Before we started with HAART, the patients CD4 cell count was 7 cells $/ \mathrm{mm}^{3}$ and his plasma HIV-1 RNA was 329.000 copies $/ \mathrm{ml}$. Thirty months later, in October 2006, patient's WBC was 1,6 x 109 /L, hemoglobin level $109 \mathrm{~g} / \mathrm{L}$ and platelet counts $28 \times 10^{9} / \mathrm{L}$. Physical examination was unremarkable. Bone marrow aspiration revealed massive infiltration of blasts, morphologically described as promyelocytes, and expressed the immunophenotypic markers of aberrant promyelocytic clone (CD15-CD117+CD34-HLD-DR-). FISH analysis of the bone marrow aspirate showed chromosomal translocation specific for APL, t(15;17), and cytogenetic analysis failed. PCR analysis detected an atypical PML/RAR $\alpha$ rearrangement of $470 \mathrm{bp}$, which was confirmed by gene sequencing. Based on these results we diagnosed APL. At the time of APL diagnosis, patient's CD4 cell count was 184 cells $/ \mathrm{mm}^{3}$ and the plasma HIV-1 RNA viral load was $<50$ copies $/ \mathrm{ml}$.

In November 2006, we started a treatment according to the standard protocol for APL. Induction therapy consisted of all-trans retinoic acid (ATRA) in dose of $45 \mathrm{mg} / \mathrm{m}^{2} /$ day taken orally for 34 days, and idarubicine in dose $12 \mathrm{mg} / \mathrm{m}^{2} /$ day intravenously through 4 days. During neutropenia treatment was complicated by sepsis. However, signs suggestive of the APL differentiation syndrome (ATRA syndrome or retinoic acid syndrome) such as pulmonary infiltrates or leukocytosis were not observed. Because of pancytopenia and the poor general condition of the patient, we interrupted HAART for a period of two months. A decrease of CD4 cell count to 47 cells $/ \mathrm{mm}^{3}$ and increase of plasma HIV-1 viral load to 394.000 copies/ml was observed in December 2006. HAART with stavudine, lamivudine and lopinavir/ritonavir was started on December 28, 2006. In February 2007, analysis of bone marrow aspirate and PCR analysis for the PML/RAR $\alpha$ indicated complete molecular remission. Given the patients poor compliance along with severe side-effects of earlier treatments and low performance status, we decided to continue consolidation treatment with solely ATRA. ATRA was given during March and April 2007 as two cycles of $45 \mathrm{mg} / \mathrm{m}^{2} /$ day for 42 days. In November 2007, we confirmed a sustained complete molecular remission. On the last follow-up in April 2008, the patient was still in complete hematological remission, and no signs of PCNSL were observed either. Plasma HIV-1 viral load was less then 50 copies of RNA per milliliter. 


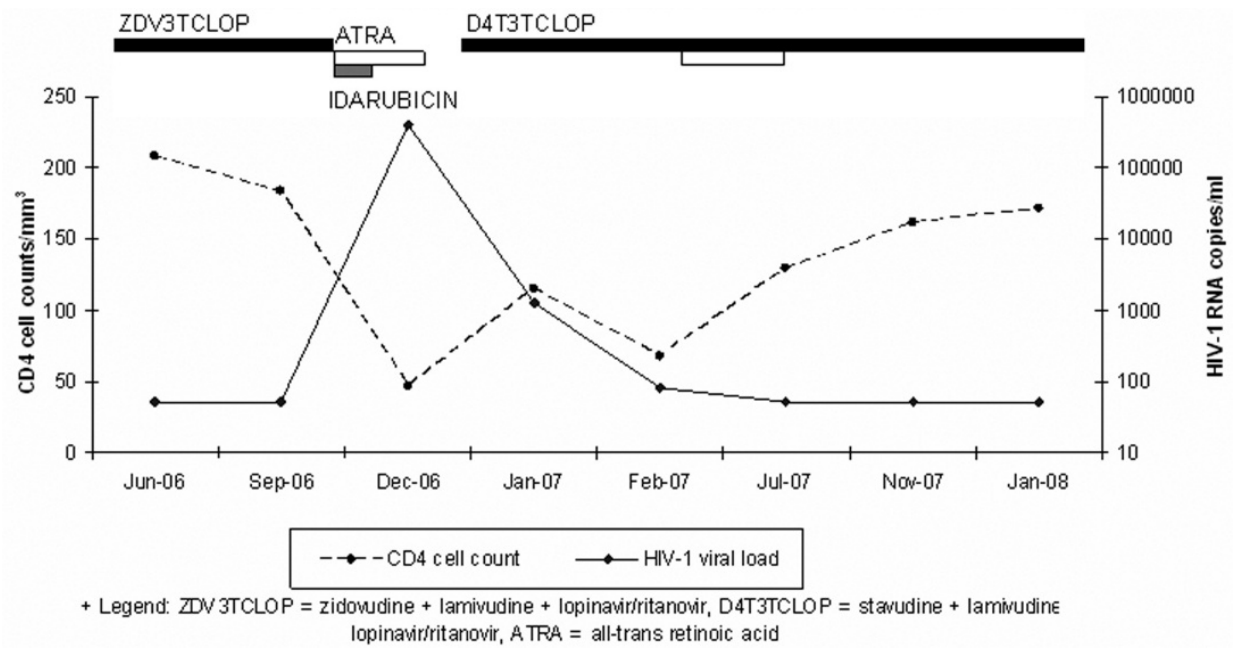

Fig. 1. The course of antiretroviral therapy, chemotherapy, CD4 cells counts and viral load measurements over time.

The course of antiretroviral treatment, chemotherapy for APL, CD4 cell counts and viral load measurements are summarized in Figure 1.

APL usually occurs de novo. However, a subgroup of patients has been described that developed APL following various chemotherapy- or radiotherapytreated malignancies [5]. An atypical PML/RAR $\alpha$ rearrangement found in our patient, and the fact that APL occurred 30 months after PCNSL, in a time frame characteristic for treatment-related APL, indicates that APL might have developed secondary to treatment of PCNSL. The treatment outcome of therapy-related APL is similar to de novo APL [5]. However, due to only five reported cases, the prognosis and course of APL in HIV-infected patients are unclear. In those reported cases, patient treated with the combined therapy of ATRA and chemotherapy entered a remission [1, 2, 3, 4, 5]. However, only two patients who concomitantly received antiretroviral therapy achieved sustained remission [2, 3]. Our patient achieved stable complete remission with induction therapy of ATRA and antracycline followed by reduced intensity consolidation therapy with ATRA, and concomitant HAART.

This therapy had acceptable side effects and induced a durable remission of APL in our patient, lasting for more than 14 months. ATRA treatment can be complicated by the APL differentiation syndrome and this diagnosis requires a high index of suspicion.

The patient's HIV-1 viral load increased transiently during interruption of HAART, but reintroduction of therapy was soon followed by a good response (Fig. 1). Based on evidence that ATRA can reduce HIV-1 viral load by acting as RT-inhibitor [6], we can speculate that it had additional positive impact in controlling HIV disease. In addition, the patient is in remission of PCNSL for more than 47 months, with no signs of major opportunistic disease and a good control of HIV plasma viral load. However, during this follow-up his CD4 cell count did not rise above $200 / \mathrm{mm}^{3}$ (Fig. 1).

In conclusion, we showed that ATRA and idarubicin based induction therapy followed by reduced intensity consolidation therapy can be applied successfully in patients with HIV-associated APL. However, the patient is still at a considerable risk for relapse and, whenever possible, standard treatment for APL to- gether with HAART should be used in HIV infected patients.

\section{REFERENCES}

1. Sutton L, Guénel P, Tanguy ML, Rio B, Dhedin N, Casassus P, Lortholary O; French Study Group on Acute Myeloid Leukaemia in HIV-Infected Patients. Acute myeloid leukaemia in human immunodeficiency virus-infected adults: epidemiology, treatment feasibility and outcome. Br J Haematol. 2001 Mar;112(4):900-8.

2. De Vita S, De Matteis S, Laurenti L, Sorà F, Tarnani M, Cingolani A, Sica S. Acute promyelocytic leukemia in an HIV-infected patient: a case report. Am J Hematol. 2006 Apr;81(4):300.

3. Kudva GC, Maliekel K, Richart JM, Batanian JR, Grosso LE, Sokol-Anderson M, Petruska PJ. Acute promyelocytic leukemia and HIV-1 infection: case report and review of the literature. Am J Hematol. 2004 Nov;77(3):287-90.

4. Calvo R, Ribera JM, Battle M, Sancho JM, Granada I, Flores A, Millá F, Feliu E. Acute promyelocytic leukemia in a HIV seropositive patient. Leuk Lymphoma. 1997 Aug;26(5-6):621-4.

5. Beaumont M, Sanz M, Carli PM, Maloisel F, Thomas X, Detourmignies L, Guerci A, Gratecos N, Rayon C, San Miguel J, Odriozola J, Cahn JY, Huguet F, Vekhof A, Stamatoulas A, Dombret H, Capote F, Esteve J, Stoppa AM, Fenaux P. Therapy-related acute promyelocytic leukemia. J Clin Oncol. 2003 Jun 1;21(11):2123-37.

6. Maeda Y, Yamaguchi T, Hijikata Y, Morita Y, Tanaka M, Hirase C, Takai S, Tatsumi Y, Kanamaru A. All-trans retinoic acid attacks reverse transcriptase resulting in inhibition of HIV-1 replication. Hematology. 2007 Jun;12(3): 263-6.

Received: September 14, 2008 / Accepted: December 3, 2008

\section{Address for correspondence:}

Ana Boban, M.D.

Division of Hematology

Department of Internal Medicine

Zagreb University School of Medicine and Clinical Hospital

Center Zagreb,

Kispaticeva 12

10000 Zagreb

Croatia

Phone: +385-98-1667808

Fax: +385-1-2421892

E-mail: ana.boban@zg.t-com.hr 